Journal of

Applied

Crystallography

ISSN 1600-5767

\title{
XRDUA: crystalline phase distribution maps by two-dimensional scanning and tomographic (micro) X-ray powder diffraction
}

\author{
Wout De Nolf, Frederik Vanmeert and Koen Janssens
}

J. Appl. Cryst. (2014). 47, 1107-1117

Copyright (C) International Union of Crystallography

Author(s) of this paper may load this reprint on their own web site or institutional repository provided that this cover page is retained. Republication of this article or its storage in electronic databases other than as specified above is not permitted without prior permission in writing from the IUCr.

For further information see http://journals.iucr.org/services/authorrights.html

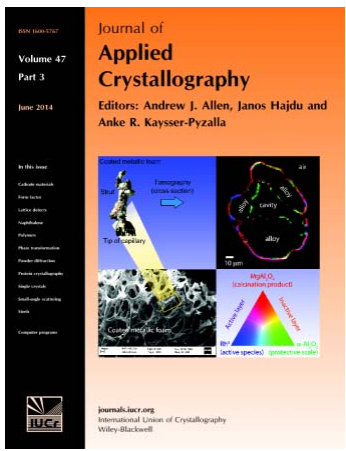

Many research topics in condensed matter research, materials science and the life sciences make use of crystallographic methods to study crystalline and non-crystalline matter with neutrons, X-rays and electrons. Articles published in the Journal of Applied Crystallography focus on these methods and their use in identifying structural and diffusioncontrolled phase transformations, structure-property relationships, structural changes of defects, interfaces and surfaces, etc. Developments of instrumentation and crystallographic apparatus, theory and interpretation, numerical analysis and other related subjects are also covered. The journal is the primary place where crystallographic computer program information is published.

Crystallography Journals Online is available from journals.iucr.org 
Journal of

Applied

Crystallography

ISSN 1600-5767

Received 4 March 2014

Accepted 11 April 2014

(C) 2014 International Union of Crystallography

\section{XRDUA: crystalline phase distribution maps by two-dimensional scanning and tomographic (micro) $\mathrm{X}$-ray powder diffraction}

\author{
Wout De Nolf,* Frederik Vanmeert and Koen Janssens \\ Department of Chemistry, University of Antwerp, Groenenborgerlaan 171, Antwerp 2020, Belgium. \\ Correspondence e-mail: woutdenolf@users.sf.net
}

\begin{abstract}
Imaging of crystalline phase distributions in heterogeneous materials, either plane projected or in virtual cross sections of the object under investigation, can be achieved by scanning X-ray powder diffraction employing X-ray micro beams and X-ray-sensitive area detectors. Software exists to convert the twodimensional powder diffraction patterns that are recorded by these detectors to one-dimensional diffractograms, which may be analysed by the broad variety of powder diffraction software developed by the crystallography community. However, employing these tools for the construction of crystalline phase distribution maps proves to be very difficult, especially when employing microfocused X-ray beams, as most diffraction software tools have mainly been developed having structure solution in mind and are not suitable for phase imaging purposes. $X R D U A$ has been developed to facilitate the execution of the complete sequence of data reduction and interpretation steps required to convert large sequences of powder diffraction patterns into a limited set of crystalline phase maps in an integrated fashion.
\end{abstract}

\section{Introduction}

Similar to the use of X-ray fluorescence spectrometry or energy dispersive X-ray spectrometry for the recording of elemental maps of heterogeneous materials (Lombi et al., 2011; Newbury \& Ritchie, 2013), X-ray powder diffraction (XRPD) can be employed for the mapping of crystalline phase distributions (De Nolf et al., 2011; Manceau et al., 2002). Although this has been technically feasible for two decades by using monochromatic pencil beams (most often at synchrotron beamlines) in combination with area detectors and motorized sample stages, relatively few studies report the practical use of this analytical method. This may be attributed to some of the limitations of XRPD imaging. The need for a focused X-ray beam geometry decreases the sensitivity of XRPD imaging (even at a synchrotron) with respect to traditional diffractometry. Furthermore, XRPD data analysis relies on the assumption that for every set of lattice planes there is an equal volume of crystallites that contribute to their diffraction, an assumption that is only met in the presence of many randomly oriented crystals ('ideal powders'). The first limitation prevents trace components from being imaged, while the second restriction limits the types of materials that can be imaged, especially with micrometre or sub-micrometre spatial resolution. The XRPD analysis tool presented in this paper greatly expands the application area of XRPD imaging by allowing 'non-ideal powders', with fewer crystallites in the X-ray beam than typically required for successful XRPD analysis, to be identified and imaged.
Two-dimensional scanning experiments, which yield planeprojected crystalline phase distributions, most often find their application in cultural heritage (Leon et al., 2010; Riekel et al., 2010; Cotte et al., 2008; Welcomme et al., 2007; Sciau et al., 2006; Lichtenegger et al., 2005; Dooryhée et al., 2005; Tamura et al., 2002; Manceau et al., 2002; Rindby et al., 1997). A summary of tomographic applications, in which crystalline phase distributions in a virtual cross section of the object under investigation were visualized, is given by ÁlvarezMurga et al. (2012). The term XRPD imaging will be used to refer to both types of investigation, since from a data analysis point of view they are very similar.

The difficulty of performing successful XRPD imaging experiments is twofold. In order to compose images of reasonable dimensions, typically thousands to tens of thousands of diffraction patterns are recorded. The identification of all crystalline phases present in such an extended series is a daunting task when dedicated analysis tools are unavailable. This is the first hurdle. Secondly, non-ideal powders (leading to Debye rings that are visibly composed of individual Bragg reflections as opposed to smooth Debye rings for ideal powders) and peak overlap often render crystalline distribution maps difficult or impossible to interpret. Most published XRPD imaging studies (not employing XRDUA) provide distribution maps on the basis of single Bragg peak intensities, which does not allow this problem to be tackled and is therefore only applicable to ideal powders. Some studies do take the entire diffraction pattern into account by combining existing data analysis tools: Palancher et al. (2011) report the 
use of the Rietveld scaling factor as a mapping quantity, Korsunsky et al. (2011) report the use of full pattern fitting for the imaging of strain in dental prostheses, and Jacques et al. (2013) report linear combination fitting of diffraction patterns and modelling of their derived pair distribution functions. Although full pattern analysis does improve treatment of nonideal powder data, $X R D U A$ provides additional features that allow for a more robust and less case-specific processing of such data.

Since the start of its development in 2004 (De Nolf \& Rickers, 2005), XRDUA has evolved to become a research tool that covers the entire data processing sequence from raw diffraction data to crystalline phase distributions. Several studies employing XRDUA have been published by the authors, most often employing XRPD with micrometre spatial resolution. In the field of cultural heritage, XRPD imaging of paint fragment cross sections allowed the description of pigment degradation pathways such as the blackening of the red pigment mercury sulfide employed by, for example, Rubens (Radepont et al., 2011) and the degradation of the yellow pigment cadmium yellow used, for example, by Ensor (Van der Snickt et al., 2009) and Van Gogh (Van der Snickt et al., 2012). Next to analysis of paint fragments on a micro scale, the use of XRPD imaging for pigment-specific hidden painting investigations has been explored (De Nolf et al., 2011). Other applications are the analysis of car paint (De Nolf \& Janssens, 2010), the characterization of catalysts used in the production of $\mathrm{H}_{2}$ from natural gas (Basile et al., 2010), uranium speciation studies (Lind et al., 2013, 2009; Denecke et al., 2008) and the characterization of monumental limestone protection treatments (Vanmeert et al., 2013). Non-affiliated studies using $X R D U A$ have been reported: the characterization of cement and cement hydration (Voltolini et al., 2013; Valentini et al., 2011; Artioli et al., 2010), partial decomposition of $\mathrm{TiH}_{2}$ (Jiménez et al., 2012), and phase transformations of zirconiabased dental prostheses (Mochales et al., 2011).

In this article, an overview will be given of the capabilities of $X R D U A$ while highlighting several critical aspects of the data analysis process of the investigations mentioned above.

\section{Targeted experiments}

A schematic representation of a typical micro-XRPD ( $\mu$-XRPD) imaging experiment at a synchrotron beamline is shown in Fig. 1. The combination of a monochromator with appropriate focusing optics (De Nolf et al., 2009) yields a monochromatic (low-divergence) pencil beam which, after diffraction from crystalline material with crystal sizes that are two or more orders of magnitude smaller than the beam size, causes Debye rings to appear on a flat X-ray-sensitive area detector. This transmission arrangement is more common than reflection geometry as the elongated footprint of the X-ray beam on the sample in reflection geometry reduces the spatial resolution with respect to transmission geometry and distorts two-dimensional scanning images. X-ray optics such as Kirkpatrick-Baez (K-B) mirror systems can compensate for this anisotropy in beam footprint at the cost of a reduced flux and therefore a reduced sensitivity with respect to a transmission geometry with the same spatial resolution. Furthermore, positioning a sample at the same distance from the detector as the calibration standard employed to determine this distance (discussed in \$3.1) is practically harder to achieve in reflection geometry than in transmission geometry. Changes in sampledetector distance make identification of crystalline phases in a diffraction pattern difficult or impossible, depending on the magnitude of the resulting shifts in Bragg peak positions. In diffraction pattern fitting, however (discussed in \$3.4), the exact sample-detector distance can be determined by $X R D U A$ for each phase, similar to the zero-shift refinement for diffractometers included in traditional Rietveld refinement software.

A two-dimensional scanning experiment consists of the stepwise movement of the object under investigation, in a plane that is (usually but not necessarily) perpendicular to the primary X-ray beam, while recording diffraction patterns after each step (Fig. 1a). Each pattern contains diffraction information of all crystalline material present in the intersection volume of the X-ray beam and the sample. The crystalline phase maps that can be reconstructed from these data therefore represent plane-projected distributions. Alternatively, the

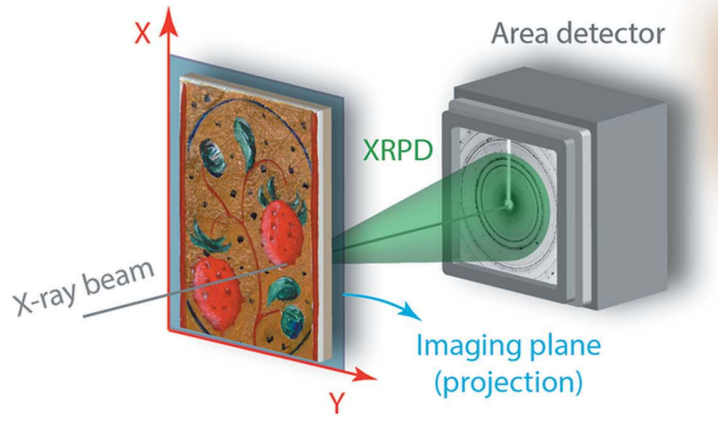

(a)

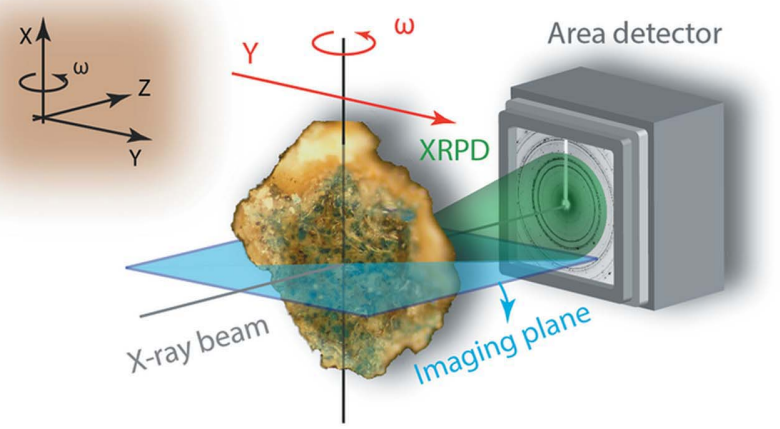

(b)

Figure 1

Two-dimensional projective $(a)$ and tomographic $(b)$ scanning XRPD experiments in transmission geometry with the primary X-ray beam along the $Z$ axis of the sample coordinate system. The aim of these experiments is to visualize crystalline phase distributions projected on the scanning plane $[X Y$ plane in $(a)$ ] or to visualize crystalline phase distributions in a virtual cross section of the object [YZ plane in $(b)]$. 
vertical translation in the scanning experiment can be replaced by a rotation around the vertical axis, i.e. the axis perpendicular to the horizontal translation direction and the primary X-ray beam (Fig. 1b). The resulting compound maps can be converted by tomographic reconstruction algorithms to maps that represent crystalline phase distributions in a virtual cross section of the sample.

\section{Data processing}

Although many functionalities of $X R D U A$ are also present in different two-dimensional diffraction pattern analysis tools and in powder diffraction analysis programs, in $X R D U A$ they are adapted specifically to the needs of XRPD imaging experiments and integrated with one another to cover the entire sequence of data transformations from raw diffraction patterns to crystalline phase distribution maps. The different steps in this process will be discussed in the order in which they are usually performed. A more detailed description can be found elsewhere (De Nolf, 2013).

\subsection{Image corrections and calibration}

The first task in processing two-dimensional diffraction patterns is to relate their pixel values to a quantity that can be theoretically described in terms of the diffracted intensity from a powder (Appendix $A$ ) and to relate their position to the geometrical variables in this theoretical description. Unwanted artefacts can be removed from the diffraction patterns manually (by masking off areas), by intensity thresholding, or by an automated process in the case of zingers (caused by radioactive decay and cosmic radiation) or saturation (occurring in CCD cameras owing to overexposure). Background and/or dark current can be removed by subtracting appropriate images or by applying a twodimensional analogue of peak stripping, an iterative filtering algorithm originally developed to describe the background in X-ray fluorescence spectra (Campbell et al., 1986). Spatial distortions in tapered fibre optic cameras can be corrected using a fiducial plate (Hammersley et al., 1996). Differences in detector pixel response can be corrected by flat field correction (Hammersley et al., 1996).

Analogously to other two-dimensional diffraction pattern analysis software, patterns of diffraction standards can be used for determining the experimental parameters that allow the assignment of scattering angle $(2 \theta), d$ spacing $(d)$ and scattering vector length $(Q)$ values to any point in the detector plane (see Appendix $B$ ). The calibration process can be based on a powder diffraction file from a database, on a list of $d$ spacings or on $d$ spacings manually assigned to selected Debye rings. These Debye rings can take the form of any conic section (ellipse, hyperbola, parabola or line). Since the position of the primary X-ray beam on the detector has been chosen as one of the calibration parameters, a detector orientation in which the detector plane is parallel to the primary X-ray beam cannot be described. Amongst the calibration parameters, two or three detector orientation para- meters are usually considered, depending on the software package used (Hammersley et al., 1996; Kumar, 2005). The three-angle description causes the calibration problem to be overdetermined so that an infinite number of solutions exist with which the standard diffraction pattern can be described. The two-angle description yields only one solution. As a consequence, however, the azimuthal angle of the diffracted radiation in the spherical coordinate system of the sample (see Fig. 6 in Appendix $A$ ) is only known up to an azimuthal shift (see Appendix $B$ ). To determine this shift, an additional parameter can be supplied in $X R D U A$ : the azimuth of the horizontal $Y Z$ plane (Fig. 1) in the detector image. At a synchrotron beamline, this plane is often the plane of the synchrotron storage ring.

\subsection{Azimuthal integration}

After image correction and calibration, the two-dimensional diffraction pattern is subjected to an additional correction (see Appendix $A$ ) whereby the recorded irradiance per pixel (SI units: $\mathrm{J} \mathrm{m}^{-2} \mathrm{~s}^{-1}$ ) is converted to radiant intensity (SI units: $\mathrm{J} \mathrm{sr}^{-1} \mathrm{~s}^{-1}$ ) by taking the distance of each detector pixel to the sample and the angle between the detector surface normal and the propagation direction of the diffracted radiation into account (see Appendix $C$ ).

Finally, the azimuthal dependency of the radiant intensity is removed by what is commonly known as azimuthal integration, to obtain a traditional diffractogram as a function of scattering angle $2 \theta$. Since not all Debye rings are fully covered by a rectangular area detector, azimuthal averaging is employed (see Appendix D). XRDUA also provides the possibility to use the azimuthal median (50\% percentile) or any other percentile instead of the average. This will discard single spots from large crystals that happen to be in Bragg orientation at certain positions and orientations in a scanning experiment, which may distort the relative Bragg peak intensities significantly. This problem is commonly encountered when employing a micrometre-sized X-ray beam to examine heterogeneous materials.

\subsection{Explorative processing}

To assist in identifying the crystalline phases present in the diffraction patterns of a two-dimensional scanning or tomographic XRPD data set and to provide immediate feedback on the data collected during an experiment, XRDUA incorporates an automated data processing mode called explorative processing. The only information needed to start such a process is the geometrical parameters determined by calibration, the settings of the required image corrections, the dimensions of the scan and the location where diffraction images will be saved (or are already present). The process converts raw two-dimensional diffraction patterns to onedimensional diffractograms (see Appendix D). Meanwhile, regions of interest (ROIs) can be selected in $2 \theta$ space in order to plot their intensity as a distribution map (see Fig. 2). Comparing different ROI maps allows for finding correlations between different Bragg peaks, although correlated maps do 
not necessarily represent the distribution of a single phase. In the painting analysis example of Fig. 2, the Bragg peaks with similar intensity distribution maps originate most likely from one pigment or ground layer material used in the painting, but not necessarily from one chemical compound; in the case of paintings, it is conceivable that pigments are mixed or degradation products formed over time. Single diffraction patterns (one-dimensional or two-dimensional) can be investigated from pixels within these ROI maps or averaged over an area determined by intensity thresholding of these maps (see Fig. 3). An alternative to the average diffraction pattern is the

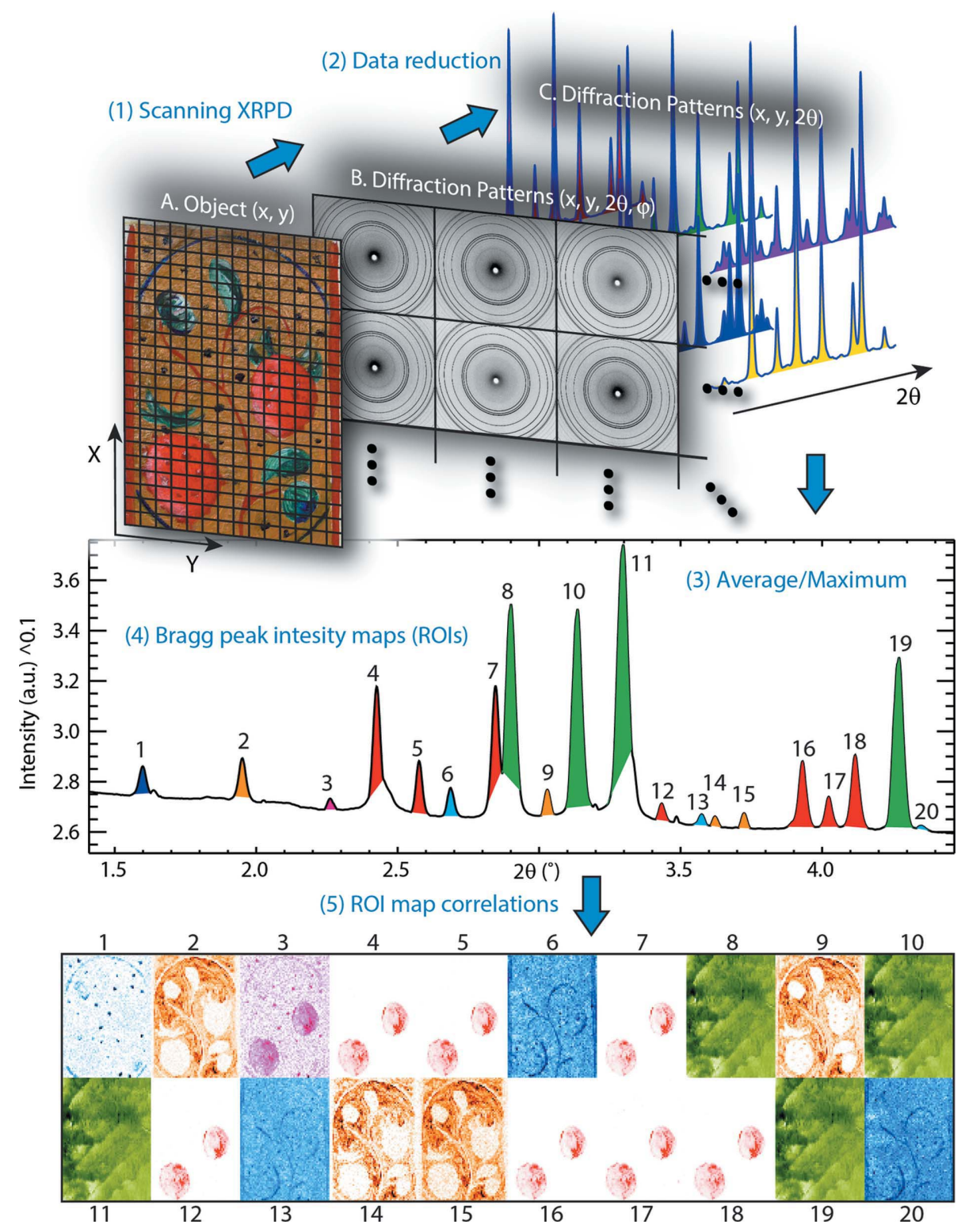

Figure 2

Explorative processing of scanning XRPD data (beamline ID15, ESRF, Grenoble, France): (1) pointwise scanning of an object through a focused X-ray beam to raster the field of view, acquiring a diffraction pattern for each pixel; (2) online correction and azimuthal integration of raw data; (3) online extraction and update of representative patterns (e.g. average or superimposed); (4) online extraction and update of Bragg peak intensity maps with linear background subtraction; (5) comparison of these maps to determine particular pixels or regions from which diffraction data should be combined (e.g. average or superimposed) for identification purposes. superimposed diffraction pattern, in which the resulting $2 \theta$-bin intensities (one-dimensional) or pixel intensities (two-dimensional) are the maximum of the $2 \theta$-bin or pixel intensities of the individual diffractions patterns. The average has the tendency to suppress the contribution of large single crystals as their diffraction spots appear and disappear in the diffraction patterns originating from different areas in the scan. This suppression is useful for the identification of fine-grained phases with the occasional larger crystals, as the relative Bragg peak intensities in the averaged diffraction pattern will be closer to the ones of an ideal powder. In contrast to the average diffraction pattern, the superimposed diffraction pattern preserves the intensities of all diffraction spots that appear in each individual diffraction pattern. This can be employed to identify coarsely grained phases as more crystal orientations are present in the superimposed diffraction pattern than in the individual diffraction patterns. As a result, the relative Bragg peak intensities of a coarsely grained phase in the superimposed diffraction pattern will be closer to the ones of an ideal powder than the relative Bragg peak intensities in individual diffraction patterns.

\subsection{Identification and modelling}

Aided by the explorative processing, a mathematical model can be constructed that will allow the automated fitting of all diffractograms in a scan by a combination of 'structural' and 'structureless' Bragg peak groups. The identification of all crystalline phases relies on powder diffraction database searches (Faber \& Fawcett, 2002) based on single diffraction patterns selected in an intelligent way from ROI maps as described above. Identified phases can be added to the model used by $X R D U A$ to fit diffractograms (see Appendix $D$ ):

$$
\begin{aligned}
& I_{\text {riet }}(2 \theta)=I_{\mathrm{bkg}}(2 \theta) \\
& +\sum_{j} S_{j} \sum_{H} F_{j H}^{2} C_{j H} \hat{\Omega}_{j}\left(2 \theta-2 \theta_{j H}\right) .
\end{aligned}
$$

This model contains a background term $I_{\mathrm{bkg}}(2 \theta)$ and a linear combination (summation over $j$ with coefficients $S_{j}$ ) of peak groups in which the shape of each peak is described by a profile function $\hat{\Omega}_{j}$. Each group $j$ contains the Bragg peaks of one crystalline phase; 
the index $H$ runs over the different diffraction peaks within a group. The factor $S_{j}$ denotes the total peak intensity scaling factor of each phase, $F_{j H}$ denotes the structure factor and $C_{j H}$ contains the part of the Lorentz-polarization factor that is not removed during azimuthal integration (see Appendix $D$ ).

(a) Identification of major phases

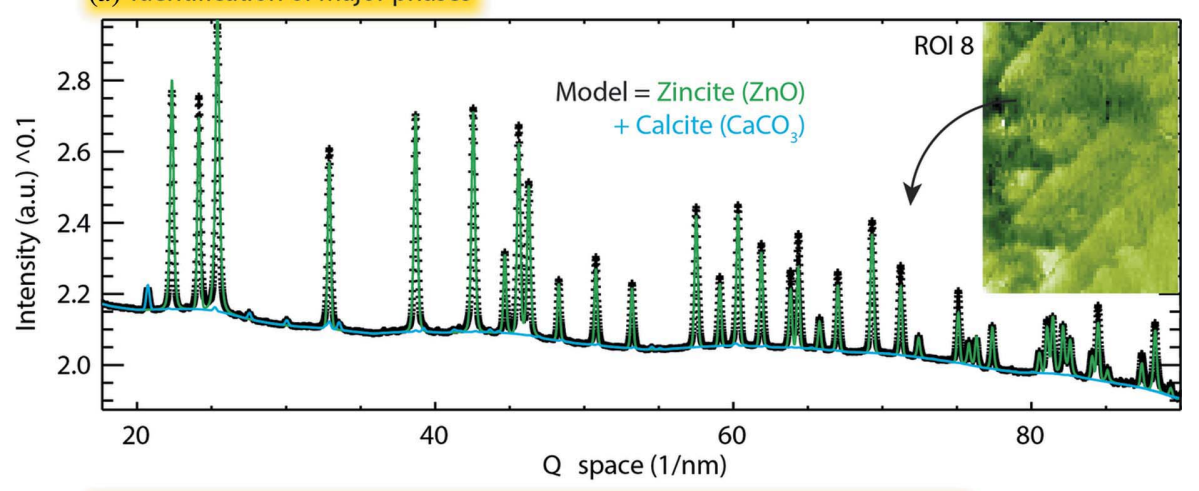

(b) Identification based on unindexed Bragg peaks from the strawberry area

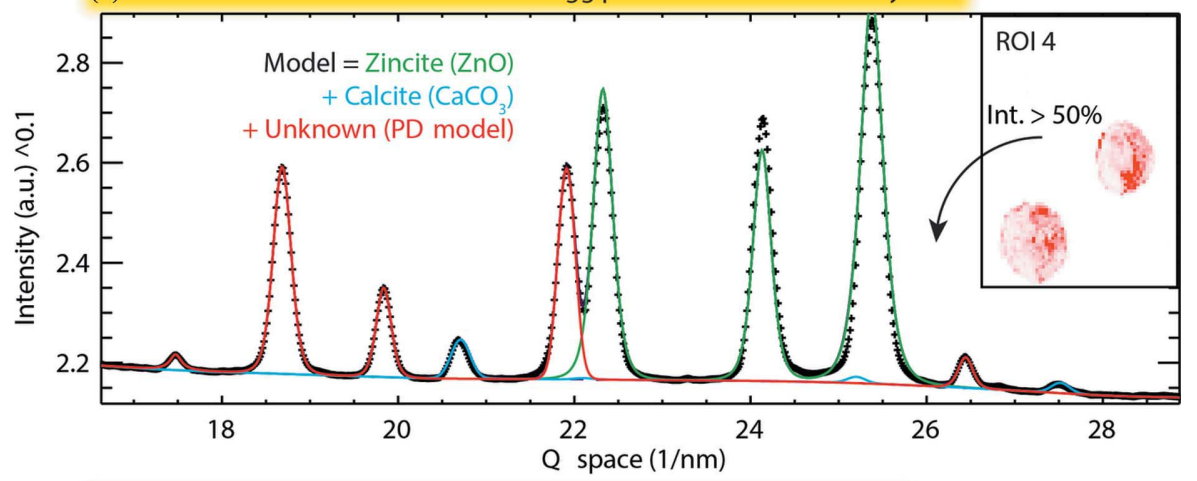

(c) Identification based on unindexed Bragg from the red markup lines
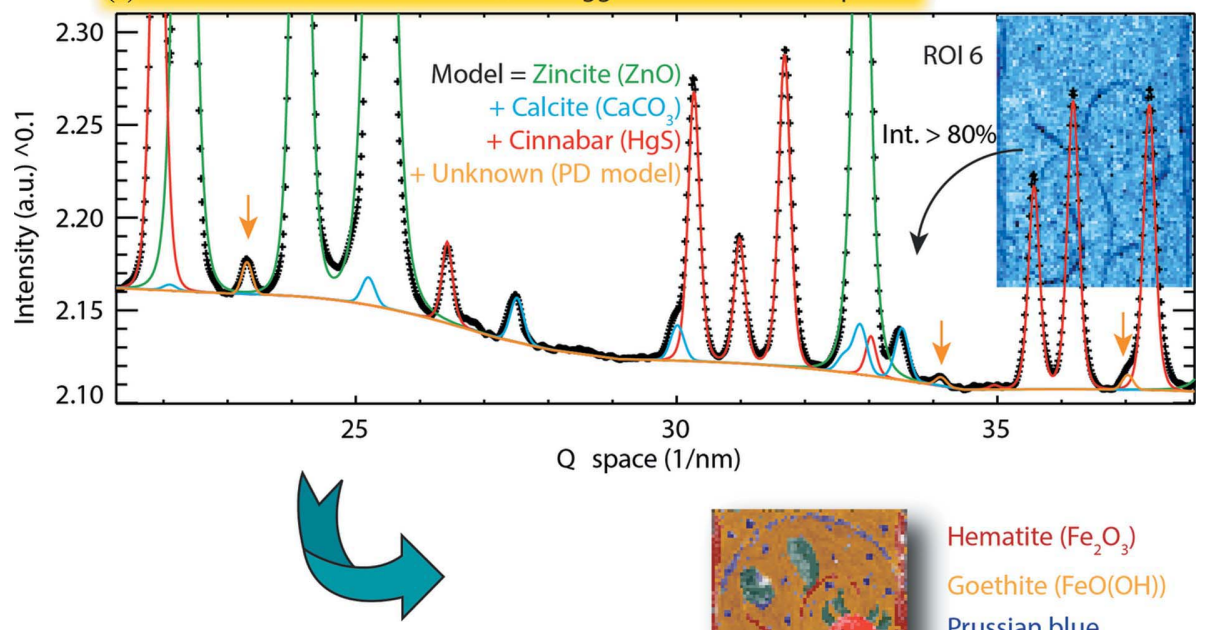

(d) Batch fitting with complete model

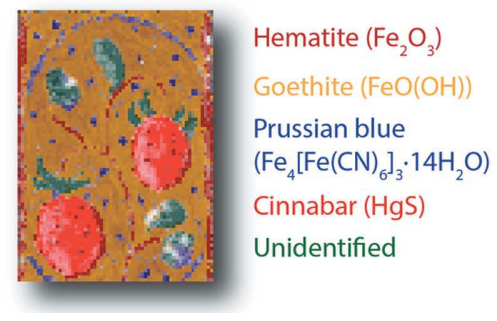

Figure 3

Fitting model construction by sequential replacement of structureless PD models by Rietveld models, based on the information presented by XRDUA after (and during) explorative processing. Data were collected at beamline ID15 (ESRF, Grenoble, France).
Peak shapes $\left(\hat{\Omega}_{j}\right)$ can be described by any of the following functions: Gaussian, Lorentzian, pseudo-Voigt, split pseudoVoigt, Thompson-Cox-Hastings pseudo-Voigt and Pearson VII. Profile shape parameters (width, mixing parameter, decay parameter etc.) can be fitted as independent parameters, or they can be described as a function of scattering angle $2 \theta$ using an empirical relation such as the well known Cagliotti peak width function. If the $\mathrm{X}$-ray source has more than one emission line, each Bragg peak in each peak group included in the model is multiplied accordingly.

When phase identification yields a complete crystal structure, peak positions $\left(2 \theta_{j H}\right)$ and relative intensities $\left(F_{j H}^{2} C_{j H}\right)$ are parameterized as a function of the sample-detector shift, the unit-cell parameters, and the fractional coordinates, site occupation factors and isotropic displacement parameters of the atoms in the asymmetric unit. The sample-detector shift is the difference between the position of the calibration standard and the position of the sample, which generally changes during a scanning experiment. The number of (non-extinct) Bragg peaks and their Miller indices are determined on the basis of a given space group setting selected from the 530 settings listed in International Tables for Crystallography (Bertaut, 2006). Parameters can be constrained during fitting by absolute box constraints (composed of the minimum lower and maximum upper values a parameter can take) or by box constraints relative to the initial value. Additionally, atomic positions can be restricted according to their Wyckoff position. The nonlinear leastsquares refinement based on a model which contains peak groups that are parameterized in this way is commonly known as Rietveld refinement; therefore a group of Bragg peaks originating from a compound with known crystal structure is referred to as a Rietveld model.

Powder diffraction databases often contain information on the space-group symmetry and the unit-cell dimensions of a compound without any atomic information. Instead, relative peak intensities are supplied. A group of Bragg peaks based on this information is referred to as a Pawley model. Since 
Bragg peaks are still determined by space-group symmetry, their positions are still parameterized by the sample-detector distance and the unit-cell parameters. On the other hand, the relative intensities (given by the product $F_{j H}^{2} C_{\mathrm{jH}}$ in the Rietveld model) are now parameterized by considering squared structure factors $F_{j H}^{2}$ as independent fit parameters and not as a function of atomic parameters. $X R D U A$ allows, however, the preservation of the relative peak intensities from the database, in which case the scaling factor $S_{j}$ is used as the only optimizable parameter to describe peak intensities, just as in a Rietveld model. A Rietveld model can always be converted to a Pawley model in those cases where the atomic parameters are considered to be fixed.

Finally, when phases cannot be identified, peaks can be grouped together on the basis of explorative processing and integrated into the model without any structural information associated. In this case, both relative intensities $\left(F_{j H}^{2}\right)$ and peak positions $\left(2 \theta_{j H}\right)$ become independent fit parameters, and the number of peaks is no longer determined by space-group symmetry. Just as the Bragg peak intensities within one group of peaks can be described by one scaling factor $S_{j}$ if desired, so can the Bragg peak positions be described by the sampledetector shift and an isotropic unit-cell deformation factor, which describes an isotropic increase or decrease in the unitcell volume without knowledge of the actual dimensions of this unit cell. A group of Bragg peaks that does not require any structural information is referred to as a pattern decomposition (PD) model. A Pawley model can always be converted to a PD model if the unit-cell parameters are known and considered to be constant.

To construct a fitting model that can describe all diffraction patterns in a scan, peak groups are sequentially added to this model until all Bragg peaks in the diffractograms extracted by explorative processing are described. During such a sequential identification process, PD groups are often used to describe those peaks that are still unidentified, after which these peak groups are exported from $X R D U A$ to be used in PDF database (http://www.icdd.com/) searching, thereby removing the peaks in the diffractogram that have already been identified and avoiding peak overlap problems. Either a list of peak positions and intensities can be extracted or the refined $2 \theta$ profile of one or more PD groups can be exported as a onedimensional diffractogram.

This iterative process is illustrated in Fig. 3 for the projective XRPD scan that has been pre-processed in Fig. 2. First the compounds that are associated with the most intense Bragg peaks in the entire map are identified (marked green in Fig. 2). By comparing the ROI intensity maps, it can be seen that the intensity distribution maps of those peaks all resemble ROI map 8. A database search of the diffraction pattern that corresponds to the most intense pixel of this map (Fig. 3a), reveals the presence of zincite $(\mathrm{ZnO})$ and a minor component (calcite, $\mathrm{CaCO}_{3}$ ). Both compounds are constituents of the ground layer of the scanned painting. To identify the other crystalline phases present, a set of Bragg peak intensity maps with different distributions is selected during the explorative processing. For each of these maps, the average of the diffraction patterns that correspond to pixels with an intensity above an appropriate threshold is calculated. In Fig. 3(b), one of the distribution maps that visualize the strawberries in the illustration is chosen (ROI map 4). The superposition of the diffraction patterns that belong to pixels with an intensity above $50 \%$ of the maximum intensity is fitted with the structural model that was constructed so far, including zincite and calcite. The unindexed Bragg peaks are added to a PD model, after which the pattern is fitted again. In this case, a database search on the fitted PD model (which can be converted to a list of $d$ spacings or a diffraction pattern, depending on what is expected by the database software) yields only one compound, namely the red cinnabar $(\mathrm{HgS})$. With a model that now contains three Rietveld models (zincite, calcite and cinnabar), the compound(s) that are distributed as shown in ROI map 6, are identified (Fig. 3c). An $80 \%$ intensity threshold is employed to calculate the representative diffraction pattern as a result of the lower signal-to-noise ratio in map 6 compared to map 4. On the basis of the small peaks that could not be fitted by the model which already includes the previously identified phases, the presence of hematite was revealed.

This iterative fitting and identification process allows for a systematic and robust construction of a model that can be used for autonomous fitting of the entire data set (see Fig. $3 d$ ). It is systematic in the sense that the entire $2 \theta$ range is scanned for distributions that would often not be discovered via the use of statistical data mining approaches such as principal component analysis (Stumpe et al., 2012). It is robust in the sense that the presence of small unidentified peaks is clearly brought to the attention of the experimentalist, minimizing the chance for minor compounds to be overlooked. While adding structures to the fitting model after identification, peak shapes and constraints of the corresponding parameters can be determined phase by phase, thereby increasing the robustness of the final model that is used to fit all measured diffraction patterns.

In the case where some phases cannot be identified [such as the green pigment in Fig. $3(d)]$, the structureless PD model remains part of the fitting model, so that at least a distribution map can be extracted from the data. The investigation of possible peak overlap, single reflections in the two-dimensional diffraction patterns and relationships between unidentified peaks are all issues that should be carefully investigated in such cases.

\subsection{Crystalline phase distribution maps}

Automatic fitting of diffraction patterns with the model described above yields a distribution map for each independent fit parameter in this model. For imaging purposes (the most common application of scanning XRPD), the maps of the total intensity scaling factors $S_{j}$ are used, since $S_{j}$ is proportional to the total volume of scattering material (provided attenuation can be neglected). Two examples of the resulting phase abundance maps are shown in Fig. 4. In the first example (Fig. 4a), the scaling factor maps of all identified pigments and grounding compounds in a physical cross section of a paint 
fragment embedded in resin allow for the visualization of the layered composition of the fragment. The same can be achieved with tomography when physical cross sectioning of the materials under investigation should be avoided (Fig. 4b). Note that the use of structureless models allows for the mapping of unidentified phases or non-Bragg diffracting phases such as the support used to fix the paint fragment for tomography in Fig. 4(b) (the red distribution, reconstructed from a PD model describing the fibre diffraction of the supporting polymer tape).

Different tomographic reconstruction algorithms are implemented in XRDUA (De Nolf, 2013) to convert $Y \omega$ scaling factor maps to $Y Z$ maps (see Fig. 1). The algebraic reconstruction technique (Kak \& Slaney, 2001), the simultaneous algebraic reconstruction technique (Kak \& Slaney, 2001) and ordered subset expectation maximization (Hudson \& Larkin, 1994), together with filtered back projection (Toft, (a) Physical cross section
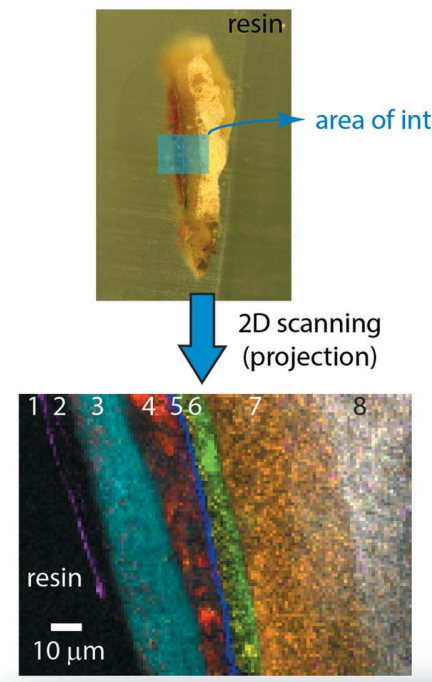

$1(, 8)$

3 Anglesite $\left(\mathrm{PbSO}_{4}\right.$

$4(, 3)$

5 Gold $(\mathrm{Au})$

6 Goethite $(\mathrm{FeO}(\mathrm{OH}))$

$6 \mathrm{H}$

$7(, 8)$

8 Gypsum $\left(\mathrm{CaSO}_{4} \cdot 2 \mathrm{H}_{2} \mathrm{O}\right)$

\section{(b) Virtual cross section}
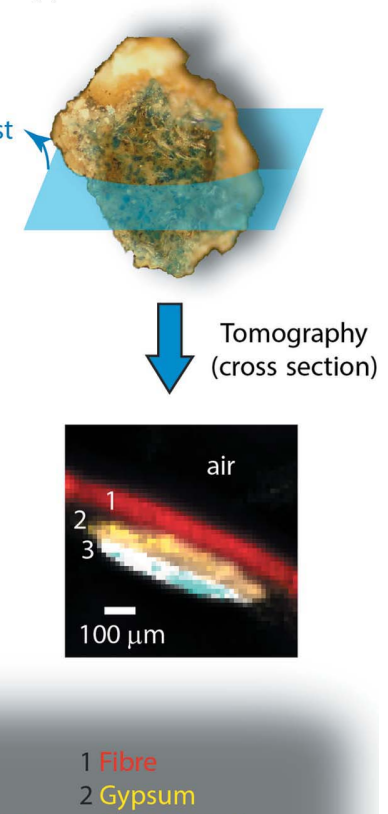

2 Gypsum

2

3 Azurite

$\left(\mathrm{Cu}_{3}\left(\mathrm{CO}_{3}\right)_{2}(\mathrm{OH})_{2}\right)$

3 Hydrocerussite

$\left(2 \mathrm{PbCO}_{3} \cdot \mathrm{Pb}(\mathrm{OH})_{2}\right)$

\section{Figure 4}

Investigation of the layered composition of two different paint fragments, the first by two-dimensional scanning of a physical cross section $(a)$ and the second by tomography of the paint fragment 'as sampled' $(b)$. The embedded paint fragment (a) was mapped at beamline PO6 (Petra III, Hamburg, Germany) with a $21 \mathrm{keV}$ X-ray beam focused by a K-B mirror system to $0.4 \times 0.4 \mu \mathrm{m}$ (horizontal $\times$ vertical). An area of $127 \times 91 \mu \mathrm{m}$ was scanned with $1 \mu \mathrm{m}$ steps, yielding 11430 diffraction patterns that were collected with $1 \mathrm{~s}$ exposures by a Pilatus $300 \mathrm{~K}$ hybrid pixel detector (Dectris). The tomography scan $(b)$ was performed at beamline $\mathrm{L}$ (HASYLAB, Hamburg, Germany) with a translation range of $750 \mu \mathrm{m}$ with $15 \mu \mathrm{m}$ step size and a rotation range of $180^{\circ}$ with $1.2^{\circ}$ step size. The $28.8 \mathrm{keV}$ X-ray beam was focused with a single-bounce elliptical capillary to $15 \times 15 \mu \mathrm{m}$. The 3111 diffraction patterns were collected with an exposure of $5 \mathrm{~s}$ by a MarCCD165 camera (Marresearch).
1996), have the advantage of fast convergence and the ability to visualize sharply aligned features, but the disadvantage of adding noise to the final distribution maps. The maximum likelihood expectation maximization algorithm (Shepp \& Vardi, 1982) and the simultaneous algebraic reconstruction technique (Kak \& Slaney, 2001) on the other hand reduce noise, are robust in the presence of artefacts in the $Y \omega$ maps (common in $\mu$-XRPD tomography, as rotation will cause some larger crystals to be oriented in Bragg orientation at some angle) but smooth out sharply aligned features (De Nolf, 2013).

As an example, the results of a tomography experiment on an Rh-coated alloy are given in Fig. 5. A metallic foam consisting of an FeCrAlY alloy was electrochemically coated with rhodium, magnesium and aluminium. The result is used as a catalyst in the production of hydrogen from natural gas (Basile et al., 2010). A single strut was isolated from the foam and subjected to XRPD tomography to study the phase composition of the coating: catalytically active $\mathrm{Rh}^{0}$ is present next to corundum $\left(\alpha-\mathrm{Al}_{2} \mathrm{O}_{3}\right)$, intended to protect the alloy from further corrosion, and a spinel phase $\left(\mathrm{MgAl}_{2} \mathrm{O}_{4}\right)$ that is a leftover product of the coating process.

\section{Installation and usage}

$X R D U A$ is written in Interactive Data Language (IDL) and is released under the GPLv3 license. Both source code and

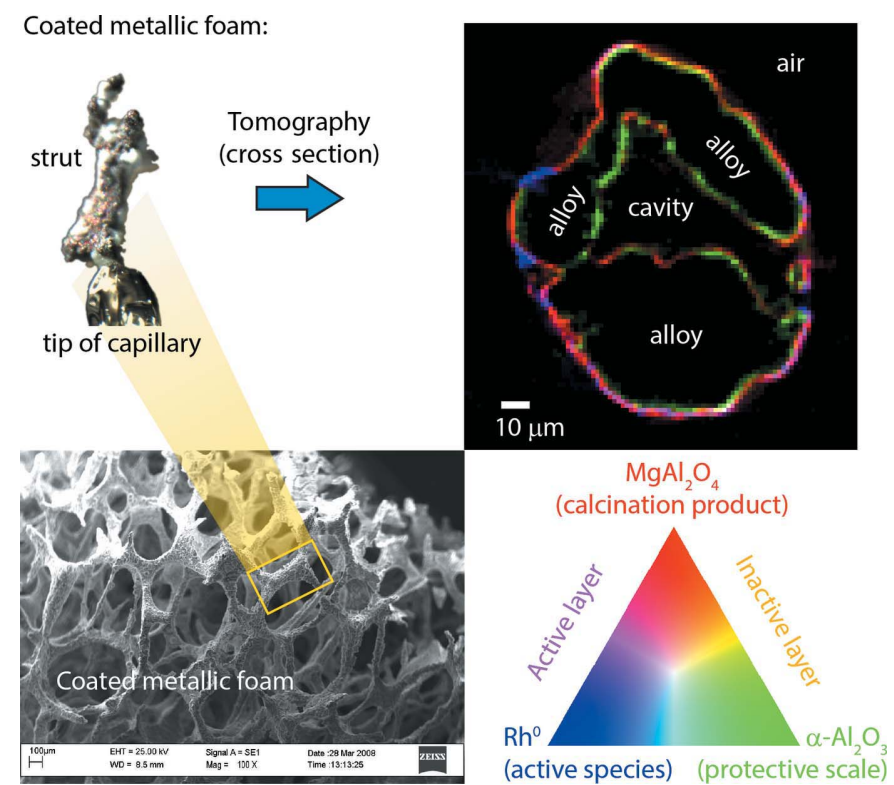

\section{Figure 5}

Characterization of the catalytic coating on a metallic foam by means of XRPD tomography. A single strut of $135 \mu \mathrm{m}$ in diameter was isolated from the foam, glued to the tip of a glass capillary and irradiated with a $18.7 \mathrm{keV}$ X-ray beam focused by a K-B mirror system to $1.9 \times 1.2 \mu \mathrm{m}$ (horizontal $\times$ vertical). The tomography experiment was performed at the MicroXAS beamline (SLS, Villigen, Switzerland) with a translation range of $180 \mu \mathrm{m}$ with $1.5 \mu \mathrm{m}$ step size (i.e. slight oversampling) and a rotation range of $180^{\circ}$ with $1.2^{\circ}$ step size. The 18271 diffraction patterns were collected with $1 \mathrm{~s}$ exposures by a Pilatus $100 \mathrm{~K}$ hybrid pixel detector (Dectris). 
executable (to be executed under the freely available IDL Virtual Machine) are available from http://xrdua.ua.ac.be/.

\section{Conclusions}

In this contribution, a tool has been presented which covers the complete data processing from two-dimensional powder diffraction patterns to crystalline phase distributions. Since $\mathrm{X}$-ray powder diffraction is a well established method, several tools already exist which cover different aspects of this process. In practice, however, the actual reconstruction of phase distributions from large numbers of diffraction patterns proves to be very difficult for complex compositions, especially on a micrometre scale. XRDUA has been shown to have the ability to solve these issues by providing a phase identification strategy and azimuthal integration and fitting methods adapted to the processing of non-ideal powder patterns, taking into account the entire diffraction pattern as opposed to single Bragg peaks.

\section{APPENDIX $A$ Diffracted intensity from a powder}

The diffracted intensity (irradiance) of a monochromatic $\mathrm{X}$-ray beam from a powder under the kinematic approximation, taking into account self-absorption for a sample with plate-like geometry (see Fig. 6) as derived by De Nolf (2013), is given by

$$
\begin{gathered}
I_{\text {diff }}(R, \varphi, 2 \theta)=I_{\mathrm{bkg}}(R, \varphi, 2 \theta) \\
+\sum_{j} \frac{S_{j}}{R^{2}} \sum_{H} F_{j H}^{2} \operatorname{LP}_{j H}(\varphi, 2 \theta) A(\varphi, 2 \theta) \hat{\Omega}_{j}\left(2 \theta-2 \theta_{j H}\right) \\
S_{j}=I_{\text {in }} \frac{r_{\mathrm{e}}^{2} \lambda^{3} S_{\text {beam }}}{8 \pi U_{j}^{2}} \frac{v_{j}}{\mu_{\mathrm{L}}}
\end{gathered}
$$

$(R, \varphi, 2 \theta)$ : spherical coordinates as defined in Fig. 6 (radius, azimuth, polar angle).

$I_{\text {diff }}$ : diffracted irradiance (SI units: $\mathrm{J} \mathrm{m}^{-2} \mathrm{~s}^{-1}$ ).

$I_{\mathrm{bkg}}$ : fluorescence or scattering not contributing to Bragg scattering (SI units: $\mathrm{J} \mathrm{m}^{-2} \mathrm{~s}^{-1}$ ).

$j$ : index loops over the crystalline phases present.

$H$ : Miller index.

$F_{j H}$ : structure factor.

$\mathrm{LP}_{j H}$ : Lorentz-polarization factor.

$A$ : attenuation factor.

$\hat{\Omega}_{j}$ : normalized peak profile as a function of scattering angle $2 \theta$ at position $2 \theta_{j H}$.

$S_{j}$ : Rietveld scaling factor of phase $j$ (SI units: $\mathrm{J} \mathrm{s}^{-1}$ ).

$I_{\text {in }}$ : irradiance at the sample of the incoming radiation (SI units: $\mathrm{J} \mathrm{m}^{-2} \mathrm{~s}^{-1}$ ).

$v_{j}$ : volume fraction of phase $j$ with respect to the total sample volume contributing to Bragg scattering.

$U_{j}$ : unit-cell volume (SI units: $\mathrm{m}^{3}$ ).
$\mu_{\mathrm{L}}$ : linear attenuation coefficient of the homogeneous platelike powder sample (SI units: $\mathrm{m}^{-1}$ ).

$r_{\mathrm{e}}$ : classical electron radius (SI units: $\mathrm{m}$ ).

$\lambda$ : wavelength of the incoming radiation (SI units: $\mathrm{m}$ ).

$S_{\text {beam }}$ : footprint area of the parallel X-ray beam with the platelike powder sample (SI units: $\mathrm{m}^{2}$ ).

In practice, the summation over $H$ is reduced owing to the presence of symmetrically equivalent reflections, thereby introducing the well known reflection multiplicity factor. The Lorentz-polarization factor for the geometry in Fig. 6 is given by (De Nolf, 2013)

$$
\begin{gathered}
\operatorname{LP}_{j H}(\varphi, 2 \theta)=\frac{P(\varphi, 2 \theta)}{\sin \theta \sin 2 \theta_{j H}}, \\
P(\varphi, 2 \theta)=1-K(\varphi)+K(\varphi) \cos ^{2} 2 \theta, \\
K(\varphi)=\frac{1-P_{\text {mono }} \cos 2 \varphi}{2}, \\
P_{\text {mono }}=\frac{s\left(1+s P_{\text {lin }}\right)-s\left|\cos ^{m} 2 \theta_{\mathrm{c}}\right|\left(1-s P_{\text {lin }}\right)}{\left(1+s P_{\text {lin }}\right)+\left|\cos ^{m} 2 \theta_{\mathrm{c}}\right|\left(1-s P_{\text {lin }}\right)} .
\end{gathered}
$$

$P$ : polarization factor.

$P_{\text {mono: }}$ part of the polarization factor depending on the monochromator, with $m=2 n$ (n: number of monochromator crystals) and $s=1$ for vertically and $s=-1$ for horizontally diffracting monochromators.

$2 \theta_{\mathrm{c}}$ : scattering angle from the monochromator crystals.

$P_{\text {lin }}$ : linear degree of polarization in the horizontal $(Y Z)$ plane of the incoming radiation.

The attenuation of the incoming and diffracted beam for a plate-like powder sample is described by the following attenuation factor (De Nolf, 2013):

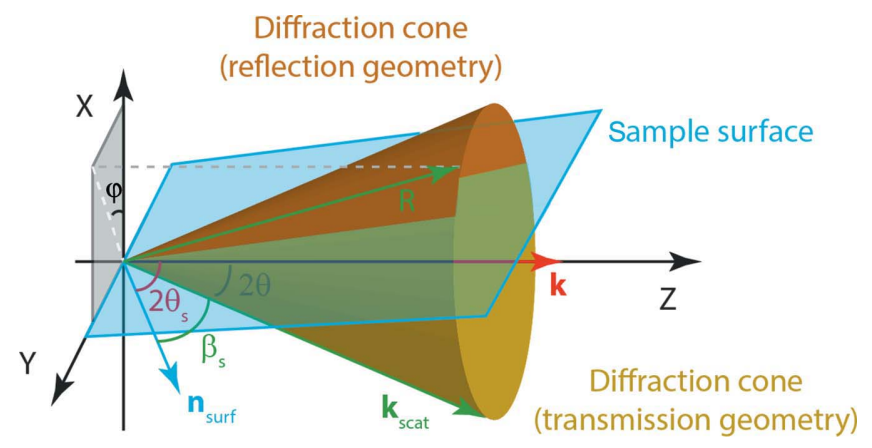

Figure 6

Diffraction from a powder sample with plate-like geometry. The incoming wavevector is denoted as $\mathbf{k}$ (red) while the diffracted wavevector is denoted $\mathbf{k}_{\text {scat }}$ (green). A point with coordinates $(x, y, z)$ in the sample coordinate system can be described in spherical coordinates $(R, \varphi, 2 \theta)$ where $\varphi$ is the azimuth and $2 \theta$ is the polar angle and also the scattering angle since $\mathbf{k}$ coincides with the $Z$ axis. Depending on $2 \theta$ and on the orientation of the surface normal $n_{\text {surf }}$, transmission geometry applies to one part of a diffraction cone (path through the sample) and reflection geometry to another part. 


$$
A(\varphi, 2 \theta)=\left\{\begin{array}{lr}
\frac{\cos \beta_{\mathrm{s}}}{\cos \beta_{\mathrm{s}}-\cos 2 \theta_{\mathrm{s}}} \exp \left(-\frac{\mu_{\mathrm{L}}}{\cos \beta_{\mathrm{s}}} x_{\mathrm{e}}\right)[1-\exp (-\chi D)] \\
\left(\cos 2 \theta_{\mathrm{s}} \neq \cos \beta_{\mathrm{s}}\right), \\
\frac{\mu_{\mathrm{L}} D}{\cos 2 \theta_{\mathrm{s}}} \exp \left(-\frac{\mu_{\mathrm{L}}}{\cos \beta_{\mathrm{s}}} x_{\mathrm{e}}\right) & \left(\cos 2 \theta_{\mathrm{s}}=\cos \beta_{\mathrm{s}}\right),
\end{array}\right.
$$

$\cos \beta_{\mathrm{s}}=\cos \left(\varphi-\varphi_{\mathrm{surf}}\right) \sin 2 \theta_{\mathrm{s}} \sin 2 \theta+\cos 2 \theta_{\mathrm{s}} \cos 2 \theta$,

$$
\chi=\mu_{\mathrm{L}}\left(\frac{1}{\cos 2 \theta_{\mathrm{s}}}-\frac{1}{\cos \beta_{\mathrm{s}}}\right) .
$$

$2 \theta_{\mathrm{s}}$ : polar angle of the surface normal of the plate-like powder sample.

$\beta_{\mathrm{s}}$ : angle between the surface normal and the scattering direction, depending on the azimuth $\varphi_{\mathrm{s}}$ of the surface normal. $D$ : thickness of the plate-like powder sample (SI units: $\mathrm{m}$ ). $x_{\mathrm{e}}$ : for parts of a diffraction cone with transmission geometry $x_{\mathrm{e}}=D$ and for parts with reflection geometry $x_{\mathrm{e}}=0$ (SI units: $\mathrm{m})$.

\section{APPENDIX $B$ Calibration}

The experimental parameters of an XRPD setup can be calculated from the Debye rings of a known powder. The detector orientation $(\alpha, \beta, \gamma)$ in the sample coordinate system (see Fig. 7) cannot be completely determined as each set of Debye rings can also originate from detector orientation $\left(\alpha^{\prime}, 0\right.$, $\gamma^{\prime}$ ), with (De Nolf, 2013)

$$
\cos \alpha^{\prime}=\cos \alpha \cos \beta, \quad \tan \gamma^{\prime}=\frac{\tan \gamma \tan \alpha+\sin \beta}{\tan \alpha-\tan \gamma \sin \beta} .
$$

The azimuth $\varphi$ of a point on an $(\alpha, \beta, \gamma)$-oriented detector plane is related to the azimuth $\varphi^{\prime}$ of an $\left(\alpha^{\prime}, 0, \gamma^{\prime}\right)$-oriented detector plane through an azimuthal shift $\delta$ :

$$
\varphi^{\prime}=\varphi+\delta, \quad \sin \delta=-\sin \beta / \sin \alpha^{\prime} .
$$

The geometric parameters of a setup in the $\left(\alpha^{\prime}, 0, \gamma^{\prime}\right)$ geometry are related to the known $d$ spacings $d_{H}$ of a diffraction standard as follows (De Nolf, 2013):

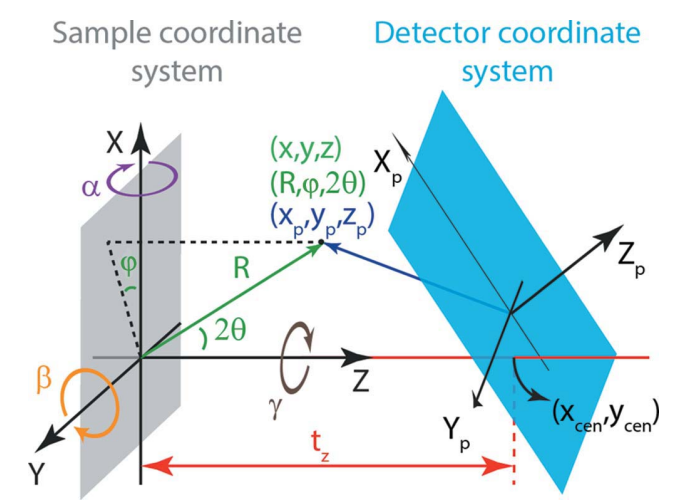

Figure 7

Relation between the sample and detector coordinate system.

$$
\begin{gathered}
\tan ^{2} 2 \theta=\left[\left(x_{\mathrm{d}} \cos \gamma^{\prime}+y_{\mathrm{d}} \sin \gamma^{\prime}\right)^{2}\right. \\
\left.+\left(-x_{\mathrm{d}} \sin \gamma^{\prime}+y_{\mathrm{d}} \cos \gamma^{\prime}\right)^{2} \cos ^{2} \alpha^{\prime}\right] \\
/\left[t_{z}-\left(-x_{\mathrm{d}} \sin \gamma^{\prime}+y_{\mathrm{d}} \cos \gamma^{\prime}\right) \sin \alpha^{\prime}\right]^{2}, \\
x_{\mathrm{d}}=p_{x}\left(x_{\mathrm{pix}}-x_{\mathrm{cen}}\right), \\
y_{\mathrm{d}}=p_{y}\left(y_{\mathrm{pix}}-y_{\mathrm{cen}}\right), \\
2 \theta=2 \arcsin \left[\lambda /\left(2 d_{H}\right)\right] .
\end{gathered}
$$

$x_{\text {pix }}, y_{\text {pix }}:$ coordinates of a point in the detector image (units: pixel).

$x_{\text {cen }}, y_{\text {cen }}:$ coordinates of the direct beam in the detector image (units: pixel).

$p_{x} \times p_{y}$ : pixel size (units: $\mathrm{m}^{2}$ pixel $^{-1}$ )

$x_{\mathrm{d}}, y_{\mathrm{d}}$ : coordinates of a point in the detector image with respect to the direct beam (SI units: $\mathrm{m}$ ).

$t_{z}$ : sample-detector distance (SI units: $\mathrm{m}$ ).

The experimental parameters that can be determined by recording a pattern from a diffraction standard are $\lambda, x_{\text {cen }}, y_{\text {cen }}$, $t_{z}, \alpha^{\prime}$ and $\gamma^{\prime}$. The parameters that need to be provided to the program are the pixel size $p_{x} \times p_{y}$ of the camera and the azimuthal shift $\delta$ (required for the polarization and attenuation factor in Appendix $A$ ). When a spatial distortion correction is applied, the pixel size is calculated from the distance between the holes in the calibration grid used for the correction (Hammersley et al., 1994; De Nolf, 2013).

\section{APPENDIX $C$ Measured intensity from a powder}

The recorded diffraction pattern is related to the diffracted irradiance (Appendix A) through the diffracted radiant intensity as follows (De Nolf, 2013):

$$
\begin{aligned}
& J_{\text {diff }}(\varphi, 2 \theta)=I_{\text {diff }}(R, \varphi, 2 \theta) R^{2} \\
& =I_{\mathrm{bkg}}(\varphi, 2 \theta) \\
& +\sum_{j} S_{j} \sum_{H} F_{j H}^{2} \operatorname{LP}_{j H}(\varphi, 2 \theta) A(\varphi, 2 \theta) \hat{\Omega}_{j}\left(2 \theta-2 \theta_{j H}\right) \\
& =I_{\text {cor }}\left(x_{\text {pix }}, y_{\text {pix }}\right) J_{\text {cor }}(\varphi, 2 \theta) \text {, } \\
& I_{\mathrm{cor}}=\frac{I_{\mathrm{det}}-I_{\mathrm{dark}}}{I_{\mathrm{ff}}-I_{\mathrm{dark}}} \\
& \begin{cases}\text { spherical : } & J_{\text {cor }}(\varphi, 2 \theta)=P_{\mathrm{ff}} /(4 \pi), \\
\text { plane : } & J_{\text {cor }}(\varphi, 2 \theta)=P_{\mathrm{ff}} t_{z}^{2} \cos ^{2} \alpha^{\prime} / \cos ^{3} \psi, \\
\text { none : } & J_{\text {cor }}(\varphi, 2 \theta)=P_{\mathrm{ff}} t_{z}^{2} \cos ^{2} \alpha^{\prime} /\left(p_{x} p_{y} \cos ^{3} \psi\right),\end{cases} \\
& \psi=\sin \alpha^{\prime} \sin 2 \theta \sin \varphi^{\prime}+\cos \alpha^{\prime} \cos 2 \theta .
\end{aligned}
$$


$P_{\text {ff }}$ (spherical): power of the spherical flood field (SI units: $\left.\mathrm{J} \mathrm{s}^{-1}\right)$.

$P_{\mathrm{ff}}$ (plane): irradiance of the flat flood field (SI units: $\left.\mathrm{J} \mathrm{m}^{-2} \mathrm{~s}^{-1}\right)$.

$P_{\text {ff }}$ (none): conversion factor (units: $\mathrm{J} \mathrm{DU}^{-1}$ ).

$\psi$ : the angle between the detector surface normal and the propagation direction of the diffracted radiation.

When using a flood field generated by a fluorescent material at the sample position (approximately spherical), the angle between the propagation direction of the diffracted beam and the detector surface normal is intrinsically taken into account when dividing by the flood field image. In the case of a plane flood field (spherical field originating from a point far away from the detector) the incident angle on the detector surface is accounted for in the $J_{\text {cor }}$ factor. However, the differences in pixel sensitivity due to a different incident angle on the detector surface are not corrected for. In the case where no flat field correction is applied, differences in pixel sensitivity are not levelled out, but the incident angle on the detector surface is still taken into account.

\section{APPENDIX $D$ Azimuthal integration}

Conversion of the recorded diffraction pattern, or more precisely the diffracted radiant intensity $J_{\text {diff }}$ as a function of azimuthal and polar angle derived from it (see Appendix $C$ ), to a one-dimensional diffractogram suitable for Rietveld refinement is achieved by inverse mapping, in which the diffraction pattern is interpolated on a $(\varphi, 2 \theta)$ grid and afterwards integrated along the azimuth $\varphi$. To facilitate the azimuthal integration, the azimuth-dependent polarization and attenuation factor are removed from $J_{\text {diff: }}$ :

$$
\begin{gathered}
J_{\mathrm{ap}}(\varphi, 2 \theta)=J_{\mathrm{diff}}(\varphi, 2 \theta) A^{-1}(\varphi, 2 \theta) P^{-1}(\varphi, 2 \theta) \sin \theta \\
=I_{\mathrm{bkg}}(\varphi, 2 \theta)+\sum_{j} S_{j} \sum_{H} F_{j H}^{2} C_{j H} \hat{\Omega}_{j}\left(2 \theta-2 \theta_{j H}\right), \\
C_{j H}=1 / \sin 2 \theta_{j H} .
\end{gathered}
$$

The part of the Lorentz term dependent on $2 \theta$ is also removed. Since the other part depends on the Bragg peak position $2 \theta_{j H}$, it is preserved in the factor $C_{j H}$. Azimuthal integration of $J_{\text {ap }}$ yields $I_{\text {riet }}$, which can be modelled using Rietveld refinement:

$$
\begin{aligned}
I_{\text {riet }}(2 \theta) & =\frac{1}{b-a} \int_{a}^{b} J_{\text {ap }}(\varphi, 2 \theta) \mathrm{d} \varphi \\
& =I_{\mathrm{bkg}}(2 \theta)+\sum_{j} S_{j} \sum_{H} F_{j H}^{2} C_{j H} \hat{\Omega}_{j}\left(2 \theta-2 \theta_{j H}\right)
\end{aligned}
$$

or in the discrete case (with equally spaced azimuthal bins)

$$
I_{\text {riet }}\left(2 \theta_{i}\right)=\frac{1}{\varphi_{n}-\varphi_{1}} \sum_{j=1}^{n} J_{\text {ap }}\left(\varphi_{j}, 2 \theta_{i}\right) \Delta \varphi=\frac{1}{n} \sum_{j=1}^{n} J_{\text {ap }}\left(\varphi_{j}, 2 \theta_{i}\right) .
$$

The last expression shows that the diffractogram $I_{\text {riet }}$ is obtained by averaging $J_{\text {ap }}$, which is sampled on a discrete $(\varphi$, $2 \theta$ ) grid, over the azimuth $\varphi$. Averaging is used instead of summation to handle Debye rings that are not completely captured by the area detector.

The authors would like to thank the synchrotron beamline staff at ID15 (ESRF, Grenoble, France), MicroXAS (SLS, Villigen, Switzerland) and PO6/BL-L (Petra III/Hasylab, Hamburg, Germany) for accommodating the experiments presented in this paper. Support from FWO 'Big Science' project $\mathrm{G} 0 \mathrm{C} 1213 \mathrm{~N}$ as well as from the BELSPO project 'S2ART' (SD/RI/04A) is acknowledged.

\section{References}

Álvarez-Murga, M., Bleuet, P. \& Hodeau, J.-L. (2012). J. Appl. Cryst. 45, 1109-1124.

Artioli, G., Cerulli, T., Cruciani, G., Dalconi, M. C., Ferrari, G., Parisatto, M., Rack, A. \& Tucoulou, R. (2010). Anal. Bioanal. Chem. 397, 2131-2136.

Basile, F., Benito, P., Bugani, S., De Nolf, W., Fornasari, G., Janssens, K., Morselli, L., Scavetta, E., Tonelli, D. \& Vaccari, A. (2010). Adv. Funct. Mater. 20, 4117-4126.

Bertaut, E. F. (2006). International Tables for Crystallography, Vol. A, 1st online ed., edited by Th. Hahn, pp. 62-76. Chester: International Union of Crystallography.

Campbell, J. L., Maenhaut, W., Bombelka, E., Clayton, E., Malmqvist, K., Maxwell, J. A., Pallon, J. \& Vandenhaute, J. (1986). Nucl. Instrum. Methods Phys. Res. Sect. B, 14, 204-220.

Cotte, M., Susini, J., Solé, V. A., Taniguchi, Y., Chillida, J., Checroun, E. \& Walter, P. (2008). J. Anal. At. Spectrom. 23, 820-828.

Denecke, M. A., De Nolf, W., Janssens, K., Brendebach, B., Rothkirch, A., Falkenberg, G. \& Noseck, U. (2008). Spectrochim. Acta Part B, 63, 484-492.

De Nolf, W. (2013). PhD thesis, University of Antwerp, Belgium, http://dx.doi.org/10067/1109100151162165141.

De Nolf, W., Dik, J., Van der Snickt, G., Wallert, A. \& Janssens, K. (2011). J. Anal. At. Spectrom. 26, 910-916.

De Nolf, W. \& Janssens, K. (2010). Surf. Interface Anal. 42, 411418.

De Nolf, W., Jaroszewicz, J., Terzano, R., Lind, O. C., Salbu, B., Vekemans, B., Janssens, K. \& Falkenberg, G. (2009). Spectrochim. Acta Part B, 64, 775-781.

De Nolf, W. J. K. \& Rickers, K. (2005). HASYLAB Jahresbericht 2004, edited by J. Schneider. HASYLAB, Hamburg, Germany.

Dooryhée, E., Anne, M., Bardiès, I., Hodeau, J. L., Martinetto, P., Rondot, S., Salomon, J., Vaughan, G. B. M. \& Walter, P. (2005). Appl. Phys. A, 81, 663-667.

Faber, J. \& Fawcett, T. (2002). Acta Cryst. B58, 325-332.

Hammersley, A. P., Svensson, S. O., Hanfland, M., Fitch, A. N. \& Hausermann, D. (1996). High Pressure Res. 14, 235-248.

Hammersley, A. P., Svensson, S. O. \& Thompson, A. (1994). Nucl. Instrum. Methods Phys. Res. Sect. A, 346, 312-321.

Hudson, H. M. \& Larkin, R. S. (1994). IEEE Trans. Med. Imaging, 13, 601-609.

Jacques, S. D. M., Di Michiel, M., Kimber, S. A. J., Yang, X., Cernik, R. J., Beale, A. M. \& Billinge, S. J. L. (2013). Nat. Commun. 4, 2536.

Jiménez, C., Garcia-Moreno, F., Rack, A., Tucoulou, R., Klaus, M., Pfretzschner, B., Rack, T., Cloetens, P. \& Banhart, J. (2012). Scr. Mater. 66, 757-760.

Kak, A. C. \& Slaney, M. (2001). Principles of Computerized Tomographic Imaging. Philadelphia: Siam. 
Korsunsky, A. M., Baimpas, N., Song, X., Belnoue, J., Hofmann, F., Abbey, B., Xie, M., Andrieux, J., Buslaps, T. \& Neo, T. K. (2011). Acta Mater. 59, 2501-2513.

Kumar, A. (2005). Report SLAC-TN-06-001. Stanford Linear Accelerator Center, Menlo Park, CA, USA, http://www.slac. stanford.edu/cgi-wrap/getdoc/slac-tn-06-001.pdf.

Leon, Y., Sciau, P., Goudeau, P., Tamura, N., Webb, S. \& Mehta, A. (2010). Appl. Phys. A, 99, 419-425.

Lichtenegger, H. C., Birkedal, H., Casa, D. M., Cross, J. O., Heald, S. M., Waite, J. H. \& Stucky, G. D. (2005). Chem. Mater. 17, $2927-$ 2931.

Lind, O. C., De Nolf, W., Janssens, K. \& Salbu, B. (2013). J. Environ. Radioact. 123, 63-70.

Lind, O. C., Salbu, B., Skipperud, L., Janssens, K., Jaroszewicz, J. \& De Nolf, W. (2009). J. Environ. Radioact. 100, 301-307.

Lombi, E., de Jonge, M. D., Donner, E., Ryan, C. G. \& Paterson, D. (2011). Anal. Bioanal. Chem. 400, 1637-1644.

Manceau, A., Tamura, N., Marcus, M. A., MacDowell, A. A., Celestre, R. S., Sublett, R. E., Sposito, G. \& Padmore, H. A. (2002). Am. Mineral. 87, 1494-1499.

Mochales, C., Maerten, A., Rack, A., Cloetens, P., Mueller, W. D., Zaslansky, P. \& Fleck, C. (2011). Acta Biomater. 7, 2994-3002.

Newbury, D. E. \& Ritchie, N. W. M. (2013). J. Anal. At. Spectrom. 28, 973-988.

Palancher, H., Tucoulou, R., Bleuet, P., Bonnin, A., Welcomme, E. \& Cloetens, P. (2011). J. Appl. Cryst. 44, 1111-1119.

Radepont, M., de Nolf, W., Janssens, K., Van der Snickt, G., Coquinot, Y., Klaassen, L. \& Cotte, M. (2011). J. Anal. At. Spectrom. 26, 959968.
Riekel, C., Burghammer, M. \& Davies, R. (2010). IOP Conf. Ser. Mater. Sci. Eng. 14, 012013.

Rindby, A., Engström, P. \& Janssens, K. (1997). J. Synchrotron Rad. 4, 228-235.

Sciau, P., Goudeau, P., Tamura, N. \& Dooryhee, E. (2006). Appl. Phys. $A, \mathbf{8 3}, 219-224$.

Shepp, L. A. \& Vardi, Y. (1982). IEEE Trans. Med. Imaging, 1, 113122.

Stumpe, B., Engel, T., Steinweg, B. \& Marschner, B. (2012). Environ. Sci. Technol. 46, 3964-3972.

Tamura, N., Celestre, R. S., MacDowell, A. A., Padmore, H. A., Spolenak, R., Valek, B. C., Meier Chang, N., Manceau, A. \& Patel, J. R. (2002). Rev. Sci. Instrum. 73, 1369-1372.

Toft, P. (1996). PhD thesis, Technical University of Denmark.

Valentini, L., Dalconi, M. C., Parisatto, M., Cruciani, G. \& Artioli, G. (2011). J. Appl. Cryst. 44, 272-280.

Van der Snickt, G., Dik, J., Cotte, M., Janssens, K., Jaroszewicz, J., De Nolf, W., Groenewegen, J. \& Van der Loeff, L. (2009). Anal. Chem. 81, 2600-2610.

Van der Snickt, G., Janssens, K., Dik, J., De Nolf, W., Vanmeert, F., Jaroszewicz, J., Cotte, M., Falkenberg, G. \& Van der Loeff, L. (2012). Anal. Chem. 84, 10221-10228.

Vanmeert, F., Mudronja, D., Fazinic, S., Janssens, K. \& Tibljas, D. (2013). X-ray Spectrom. 42, 256-261.

Voltolini, M., Dalconi, M. C., Artioli, G., Parisatto, M., Valentini, L., Russo, V., Bonnin, A. \& Tucoulou, R. (2013). J. Appl. Cryst. 46, 142-152.

Welcomme, E., Walter, P., Bleuet, P., Hodeau, J., Dooryhee, E., Martinetto, P. \& Menu, M. (2007). Appl. Phys. A, 89, 825-832. 